\title{
Knockdown of Angiopoietin-Like Protein 2 Ameliorates Diabetic Nephropathy by Inhibiting TLR4
}

\author{
Suxia Yang Junwei Zhang Shiying Wang Jun Shi Xinxin Zhao \\ Department of Nephrology, Huaihe Hospital of Henan University, Kaifeng, China
}

Key Words

Angpt|2 $\bullet$ TLR4 $\bullet$ Diabetic nephropathy $\cdot$ Podocyte $\bullet$ Inflammatory cytokines $\bullet$ Renal injury

\begin{abstract}
Background/Aims: Angiopoietin-like protein 2 (ANGPTL2) was reported to be implicated in the pathogenesis of inflammatory disease. Its role in diabetic nephropathy (DN) remained illdefined. Methods: qRT-PCR and western blot analysis were performed to detect the expressions of ANGPTL2 or TLR4 in streptozotocin (STZ)-induced DN rats and HG-stimulated podocytes. The renal injury index including 24-h proteinuria, blood glucose level, serum creatinine and blood urea nitrogen were measured in DN rats using corresponding commercial kits. The effect of ANGPTL2 knockdown on the secretion or expression of inflammatory cytokines was detected by ELISA or qRT-PCR analysis. The effect of ANGPTL2 knockdown on extracellular matrix (ECM) accumulation was determined by testing TGF- $\beta 1$, Collagen-IV, fibronectin (FN) and PTEN expression via western blot. Results: ANGPTL2 and TLR4 were both highly expressed in DN rats compared with control group. ANGPTL2 knockdown alleviated renal injury in STZinduced DN rat model. ANGPTL2 knockdown also suppressed inflammatory cytokines (IL6 , TNF- $\alpha$, MCP-1, IL-1 $\beta$ ) expression and ECM accumulation (TGF- $\beta 1$, Collagen-IV, FN, PTEN) in HG-induced podocytes. Moreover, ANGPTL2 knockdown led to a significant decrease of TLR4 expression in both DN rat and cell model. Furthermore, TAK-242 treatment exacerbated the inhibitory effect of ANGPTL2 knockdown on inflammatory cytokines expression and ECM accumulation in HG-induced podocytes. Conclusion: ANGPTL2 knockdown ameliorates DN by inhibiting TLR4 expression, an observation contributing to a better understanding of DN pathogenesis.

C 2017 The Author(s)

Published by S. Karger AG, Basel
\end{abstract}

\section{Introduction}

Diabetic nephropathy (DN) is one of the leading microvascular complications in patients with diabetes, affecting approximately $15-25 \%$ of type 1 diabetes patients and $30-40 \%$ of type 2 diabetes patients [1]. Therefore, it is not surprising that DN is a major S. Yang and J. Zhang contributed equally to this work. 


\section{Cellular Physiology Cell Physiol Biochem 2017;43:685-696

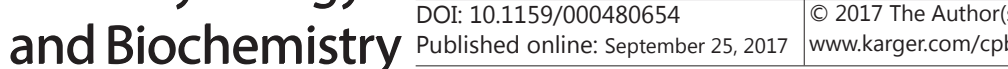

Yang et al.: ANGPTL2 Knockdown Ameliorates Diabetic Nephropathy

cause of chronic renal failure in recent years [2]. The clinical and pathological features of early DN include deposition of extracellular matrix (ECM) proteins in the mesangium, glomerular basement membrane (GBM) thickening, tubulointerstitial fibrosis as well as the presence of albuminuria, eventually resulting in progressive renal injury [3]. Inflammatory processes facilitated by innate immune responses are considered to play a pivotal role in the development and progression of DN [4]. Currently, it is convincingly demonstrated that the pathogenesis of DN is complex and involved in many aspects, including hyperglycemia, genetic factor and disorder of biochemical metabolism [5]. Hyperglycemia can activate various intracellular signaling factors including transforming growth factor (TGF)- $\beta 1$, leading to the accumulation of ECM [6]. In order to search for new therapeutic strategies for DN management, the research on DN etiology is of great significance.

Angiopoietin-like protein 2 (ANGPTL2), belonging to a family of secreted proteins structurally similar to angiopoietins, is characterized by an $\mathrm{N}$-terminus coiled-coil domain and a C-terminus fibrinogen-like domain [7]. ANGPTL2 has been demonstrated to be a crucial regulator of inflammation in adipose tissue via regulating inflammatory signaling in endothelial cells and systemic insulin resistance in diet-induced obese mice [8,9]. ANGPTL2 is documented to be implicated in the pathogenesis of inflammatory disease such as diabetes, heart failure and atherosclerosis $[8,10,11]$. ANGPTL2 is highly expressed in the glomeruli from patients with DN but absent in normal glomeruli of control kidney [12]. In addition, upregulation of ANGPTL2 in diabetic glomerulopathy is mainly distributed in podocytes and involved in the progression of diabetic glomerulopathy [12]. However, the biological role of ANGPTL2 and its underlying mechanism in DN remains unclear.

Toll-like receptors (TLRs), important regulators of innate immune system, are abundantly expressed in immune cells, including macrophages, dendritic cells, lymphocytes, and nonimmune cells (such as kidney tubular epithelial cells, endothelial cells and podocytes) [13]. TLRs play a fundamental role in the activation of innate and adaptive immune responses by triggering a signaling cascade via release of several inflammatory cytokines [14]. It is well established that up-regulation of TLR4 is observed in patients with diabetic microvascular disease, particularly in nephropathy, and is related to increased release of various biomediator such as interleukin-6 (IL-6), tumor necrosis factor- $\alpha$ (TNF- $\alpha$ ), monocyte chemotactic protein-1 (MCP-1), and IL-1 $\beta[15,16]$. TLR4 has been previously revealed to contribute to the development of inflammation-associated renal injury and kidney disease including DN $[16,17]$. More importantly, TLR4 was hypothesized to be a potential receptor for ANGPTL2 in end othelial cells and monocytes by a previous study [18]. However, the detailed molecular association between ANGPTL2 and TTLR4 remains to be further confirmed in DN.

In our study, we explored the role of ANGPTL2 and its underlying mechanism in DN by establishing streptozotocin (STZ)-induced DN rats as in vivo models of DN and high glucoseinduced podocytes as in vitro models of DN.

\section{Materials and Methods}

\section{Animal model and treatment}

Forty specific pathogen-free (SPF) grade male Sprague-Dawley rats (aged 12 weeks and weighing 200 to $250 \mathrm{~g}$ ) were purchased from Shanghai Laboratory Animal Center (Shanghai, China). The rats were housed in an SPF lab with constant temperature $\left(21 \pm 2^{\circ} \mathrm{C}\right)$, constant humidity $(55 \pm 2 \%)$ and an alternating $12 \mathrm{~h}$ light/dark cycles with free access to normal basal diet and water. Randomly, these rats were divided into four groups $(\mathrm{n}=10)$ : control group, DN group, DN + sh-control group and DN + sh-ANGPTL2 group. The rat models of DN were established by intraperitoneally injection of STZ (dissolved in $0.1 \mathrm{M}$ citrate acid buffer, $\mathrm{pH}$ = 4.0; Sigma, St. Louis, MO, USA) at $50 \mathrm{mg} / \mathrm{kg}$. The control rats were intraperitoneally administrated with an equal amount of citric acid buffer. Blood glucose levels were measured on the third day after STZ injection. In addition, the DN rats in DN + sh-ANGPTL2 group or DN + sh-control group received an intravenous injection of $100 \mu \mathrm{l}$ adenovirus $\left(\sim 1 \times 10^{5} \mathrm{IU} / \mu \mathrm{l}\right)$ carrying shRNA against ANGPTL2 (sh-ANGPTL2) or scrambled shRNA (sh-control) one week after STZ injection. Animals with blood glucose levels over $16.7 \mathrm{mmol} / \mathrm{L}$ for 


\section{Cellular Physiology Cell Physiol Biochem 2017;43:685-696 \begin{tabular}{c|c|c|c|}
\hline DOI: 10.1159/000480654 & ( ) 2017 The Author(s). Published by S. Karger AG, Basel
\end{tabular} and Biochemistry Published online: September 25, 2017 www.karger.com/cpb}

Yang et al.: ANGPTL2 Knockdown Ameliorates Diabetic Nephropathy

3 continuous examinations were considered as successful DN modeling. The rats were euthanatized at 12 weeks after STZ injection and the renal tissues were removed and frozen at $-80^{\circ} \mathrm{C}$ for next analysis.

\section{Biochemical determination}

The body weight was measured after the rats were sacrificed at the end of the $12^{\text {th }}$ weeks after modeling. Blood samples were collected from the abdominal arota into non-hepatinised tubes, allowed to clot for $2 \mathrm{~h}$ at room temperature and then centrifuged at $2000 \times \mathrm{g}$ for 10 minutes. Blood glucose, serum creatinine, as well as blood urea nitrogen levels were measured using corresponding commercial assay kits (Jiancheng Biotech, Nanjing, China) following the manual instructions on an automatic biochemical machine (Toshiba 7170, Tokyo, Japan). 24-h proteinuria was detected by Bradford Protein Assay Kit (Bio-Rad, Hercules, CA, USA).

\section{Preparation of primary rat podocytes}

SD rats were euthanized using an excess amount of pentobarbital (over $50 \mathrm{mg} / \mathrm{kg}$ body weight, intravenous injection) under inhalation anesthesia with $2 \%$ isoflurane. Kidneys were immediately excised and perfused with a mixture of Dynabeads $(\varphi 4.5 \mu \mathrm{m})$ and iron powder $(\varphi 6 \mu \mathrm{m})$. Renal cortical tissue was then cut into 1-2 mm cubes in Hanks' balanced salt solution (HBSS; Invitrogen, Carlsbad, CA, USA), digested and then passed through a sieve with $200 \mu \mathrm{m}$ diameters (Thermo Fisher Scientific, Basingstoke, UK). The glomeruli after magnetic treatment were suspended in a suitable amount of HBSS and then passed through a smaller 70- $\mu$ m cell strainer (BD Biosciences, Stockholm, Sweden). The glomeruli retaining on the top of $70 \mu \mathrm{m}$ cell strainer were carefully collected. The glomeruli and outgrowing podocytes were trypsinized and passed through a $25 \mu \mathrm{m}$ sieve to separate podocytes from glomerular cores. Podocyte phenotypes were confirmed using podocyte-specific antibodies against Wilms' tumor-1 protein (WT-1; Santa Cruz Biotechnology, Santa Cruz, CA, USA) and podoxin (Santa Cruz Biotechnology).

\section{Podocyte culture and treatment}

Conditionally immortalized mouse podocytes were cultured in RPMI 1640 medium (Gibco Lab., Grand Island, NY, USA), supplemented with 10\% fetal bovine serum (FBS; Gibco Lab.), $100 \mathrm{U} / \mathrm{ml}$ penicillin G/ streptomycin, $5.6 \mathrm{mM}$ glucose (Dingguo Changsheng Biotechnology Co., Ltd., Beijing, China) and $10 \mathrm{U} / \mathrm{ml}$ recombinant mouse interferon- $\gamma$ (IFN- $\gamma$; Sigma). To knockdown ANGPTL2, podocytes were transfected with siRNA against ANGPTL2 (si-ANGPTL2) or siRNA negative control (si-NC) using Lipofectamine 2000 (Invitrogen). Subsequently, podocytes were treated with $30 \mathrm{mM}$ D-glucose (high glucose, HG) for $24 \mathrm{~h}$ to induce cell model of DN. Also, $1 \mu \mathrm{M}$ TAK-242 was used to suppress TRL4 expression in podocytes.

\section{Quantitative real-time PCR ( $q$ RT-PCR)}

Total RNA from kidney cortex and podocytes was isolated using Trizol RNA isolation system (Invitrogen) and reversely transcribed into cDNAs using the SuperScriptTM III First-Strand Synthesis System (Invitrogen). For detection of ANGPTL2 and TLR4, qRT-PCR was carried out in an ABI PRISMs 7000 Sequence Detection System (Applied Biosystems Japan, Tokyo, Japan) with SYBR Green Master Mix (Roche Applied Science, Mannheim, Germany) according to the manufacturer's instructions. The PCR program was performed based on the conditions listed below: $95^{\circ} \mathrm{C}$ for $10 \mathrm{~min}$, followed by 40 cycles of $95^{\circ} \mathrm{C}$ for $15 \mathrm{~s}$ and $60^{\circ} \mathrm{C}$ for $1 \mathrm{~min}$. The PCR results were calculated using the $2^{-\Delta \Delta C \mathrm{~T}}$ method as described previously [19] and normalized to GAPDH expression.

\section{Western blot analysis}

Kidney cortex or cultured podocyte lysates were prepared using RIPA extraction buffer (Beyotime, Haimen, China). Equal amounts of cell lysates containing $30 \mu \mathrm{g}$ of protein were loaded onto $10 \%$ sodium dodecyl sulfate-polyacrylamide electrophoresis gels (SDS-PAGE) for separation and then transferred to polyvinylidene fluoride membranes (PVDF; Millipore, Billerica, MA, USA). Following blocked by $5 \%$ nonfat dry milk for $1 \mathrm{~h}$ at room temperature, membranes were incubated overnight at $4^{\circ} \mathrm{C}$ with the primary antibodies against ANGPTL2, TLR4, TGF- $\beta 1$, PTEN, Collagen IV, FN or $\beta$-actin 1:1000; Abcam, Cambridge, MA, USA). $\beta$-actin was used as the internal control. The membranes were further probed with horseradish peroxidase-conjugated anti-rabbit IgG (1:2000; Santa Cruz, Waltham, MA, USA) for $2 \mathrm{~h}$ at room temperature. Proteins bands were visualized by enhanced chemiluminescence reagents (Millipore, Plano, TX, USA). 


\section{Cellular Physiology Cell Physiol Biochem 2017;43:685-696 \begin{tabular}{l|l|l} 
DOI: 10.1159/000480654 & (c) 2017 The Author(s). Published by S. Karger AG, Basel
\end{tabular} and Biochemistry Published online: September 25, 2017 www.karger.com/cpb}

Yang et al.: ANGPTL2 Knockdown Ameliorates Diabetic Nephropathy

Enzyme-linked immunosorbent assay (ELISA)

The concentrations of serum inflammatory cytokines IL-6, TNF- $\alpha$, MCP-1 and IL-1 $\beta$ levels in blood and kidney cortex of STZ-induced DN rats were determined by a specific ELISA kit (R\&D Systems, Minneapolis, MN, USA) according to the manufacturer's instructions.

\section{Histopathological examination and immunofluorescence analysis}

Renal tissues were fixed in $4 \%$ paraformaldehyde for $24 \mathrm{~h}$, processed conventionally, embedded in paraffin, and sectioned at $5 \mu \mathrm{m}$. Sections were fixed to glass slides and stained with Weigert's hematoxylin solution for $10 \mathrm{~min}$, rinsed in distilled water for $5 \mathrm{~min}$, stained with Masson ponceau acid fuchsin solution for $10 \mathrm{~min}$, washed with distilled water for $2 \mathrm{~min}$, placed in 1\% phosphomolybdic acid solution for $5 \mathrm{~min}$, and stained with aniline blue solution for $5 \mathrm{~min}$. The Masson trichrome-stained slides were then digitized with optical microscope ( $\times 400$ magnification). 10 Renal sections from rats in each group $(\mathrm{n}=10)$ were immunostained with primary antibodies against WT1 (Santa Cruz Biotechnology). The slides were then stained with Cy2-conjugated immunoglobulin secondary antibody (Jackson ImmunoResearch Laboratories, West Grove, PA, USA). Ten glomeruli were randomly examined in each section under a laser scanning microscopy (400x magnification; Leica Microsystems, Wetzlar, Germany) and the average in each group was recorded.

\section{Statistical analysis}

All data were expressed as mean \pm standard deviation (SD) and analyzed using SPSS 16.0 software (SPSS, Inc., Chicago, IL, USA). The statistical significance was calculated using an unpaired Student's $t$-test and two way analysis of variance (ANOVA) with Bonferroni test. $P<0.05$ was considered statistically significant.

\section{Results}

\section{ANGPTL2 and TLR4 were both up-regulated in DN rats}

To examine the role of ANGPTL2 and TLR4 in the development of DN, the expressions of ANGPTL2 and TLR4 at mRNA and protein levels in renal tissues of DN rats were analyzed by qRT-PCR and western blot, respectively. The results showed that ANGPTL2 and TLR4 were both highly expressed at mRNA (Fig. 1A) and protein (Fig. 1B) levels in DN rats when compared to control group.

Fig. 1. Expression levels of ANGPTL2 and TLR4 in DN rats. The expression levels of ANGPTL2 and TLR4 in control rats and $\mathrm{DN}$ rats were evaluated by qRT-PCR (A) and western blot (B). The data was expressed as mean $\pm \mathrm{SD}, \mathrm{n}$ $=6, * \mathrm{P}<0.05$. Unpaired $\mathrm{t}$ test was performed.

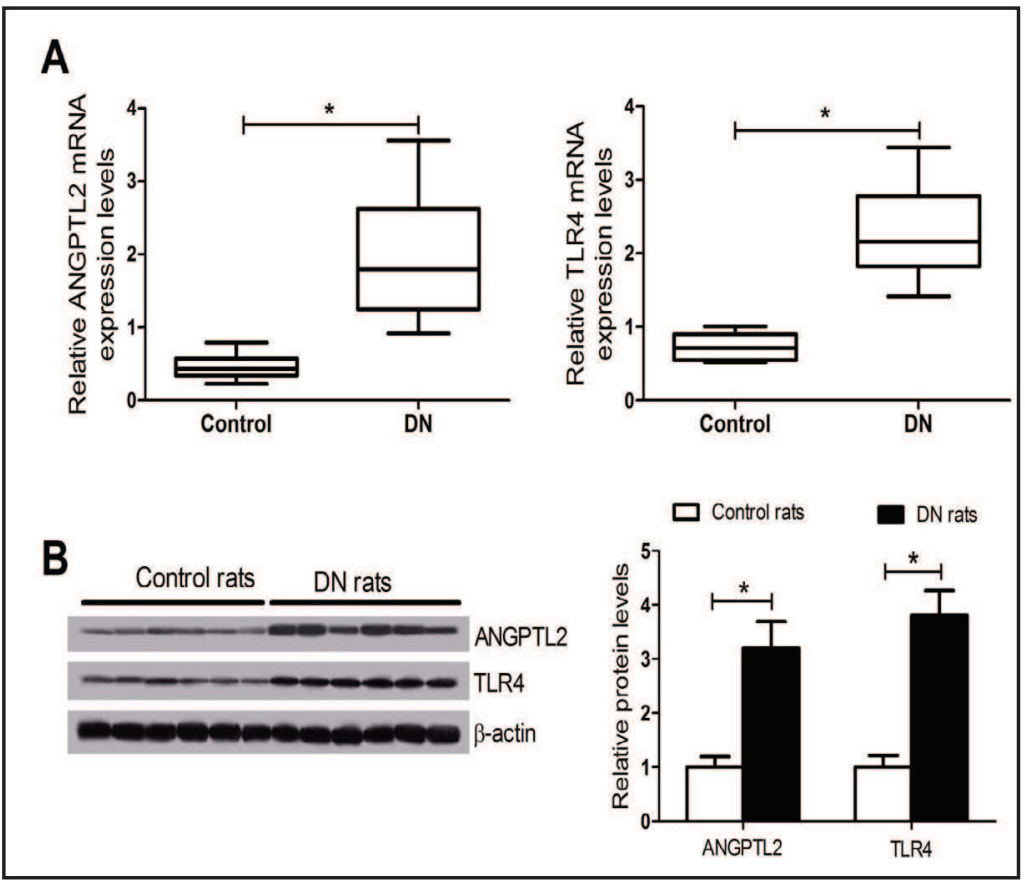




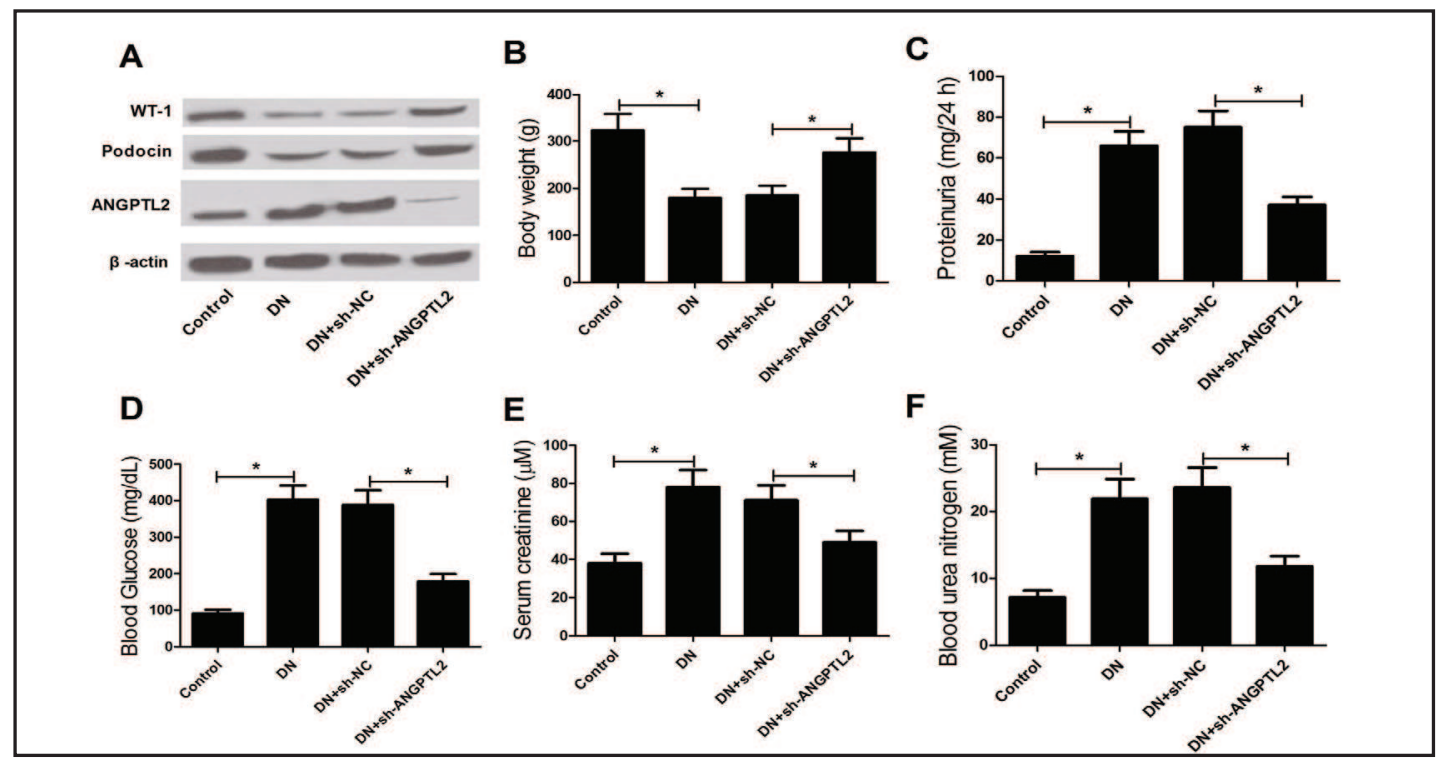

Fig. 2. Effect of ANGPTL2 knockdown on renal damage in STZ-induced DN rats. (A) The expression of ANGPTL2 and podocyte markers WT-1 and podocin was determined in control rats, DN rats, or DN rats infected with sh-ANGPTL2 or sh-NC using western blot. The body weight (B) and the concentrations of 24-h proteinuria (C), blood glucose level (D), serum creatinine (E) and blood urea nitrogen (F) were examined in control rats, DN rats, or DN rats infected with sh-ANGPTL2 or sh-NC. The data was expressed as mean \pm SD, $\mathrm{n}=3,{ }^{*} \mathrm{P}<0.05$. Two-way ANOVA with Bonferroni test was used.

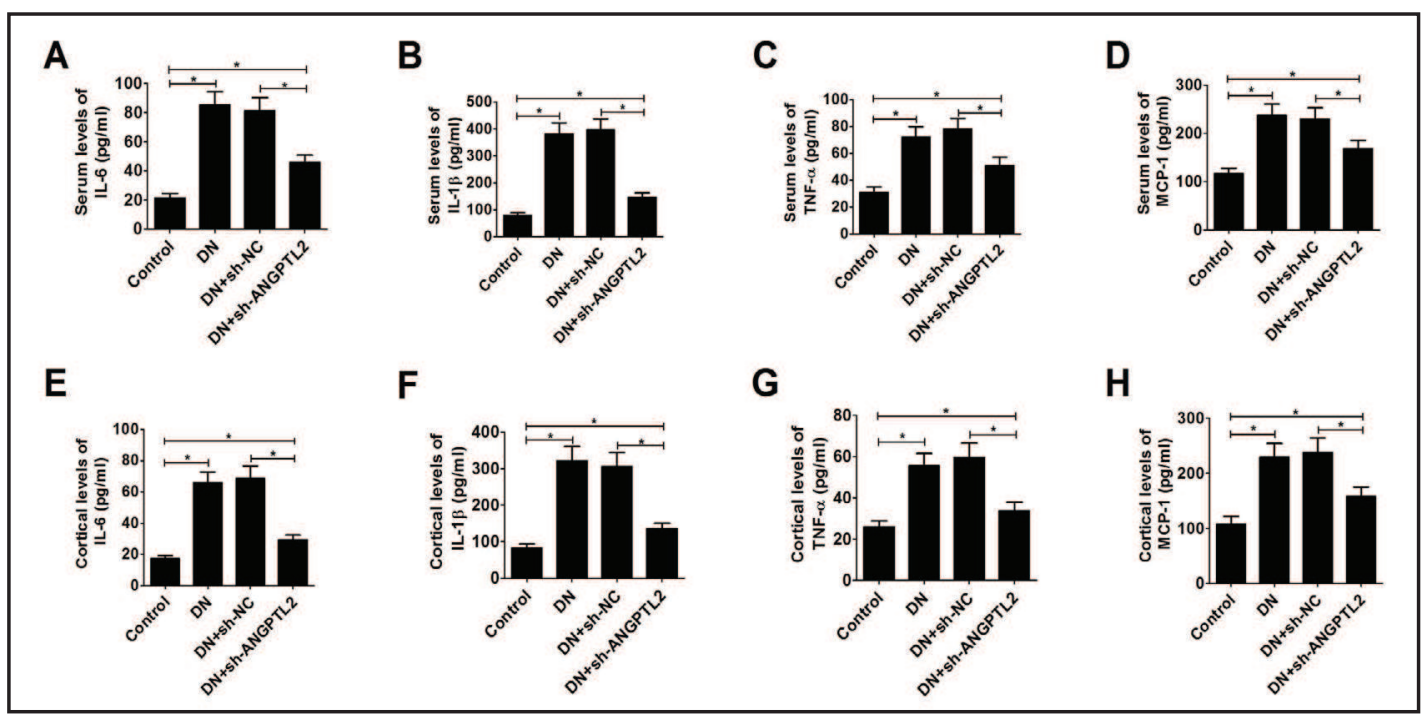

Fig. 3. Effect of ANGPTL2 knockdown on the secretion of inflammatory cytokines in DN rats. The concentrations of IL-6 (A and E), IL-1 $\beta$ (B and F), TNF- $\alpha$ (C and G), and MCP-1 (D and H) in blood and kidney cortex of $\mathrm{DN}$ rats were measured by ELISA analysis. The data was expressed as mean $\pm \mathrm{SD}, \mathrm{n}=3,{ }^{*} \mathrm{P}<0.05$. Two-way ANOVA with Bonferroni test was used.

\section{ANGPTL2 knockdown alleviated STZ-induced renal damage of DN rats}

To confirm the biological role of ANGPTL2 in the development of DN, DN rats received an intravenous injection of adenovirus carrying sh-ANGPTL2 or shRNA. Firstly, western blot analysis revealed that ANGPTL2 expression was obviously decreased in the sh-ANGPTL2 group, suggesting the successful knockdown of ANGPTL2 (Fig. 2A). Moreover, podocyte 


\section{Cellular Physiology Cell Physiol Biochem 2017;43:685-696 \begin{tabular}{l|l|l}
\cline { 1 - 1 } DOI: 10.1159/000480654 & ( ) 2017 The Author(s). Published by S. Karger AG, Basel
\end{tabular}

Fig. 4. Effect of ANGPTL2 knockdown on renal histology and ECM accumulation in DN rats. (A) Representative images $(\times 400$ magnification) of light microscopy for kidney sections stained with Masson's trichrome and quantification of Masson's trichrome-positive area in control rats, DN model rats, or DN model rats infected with sh-ANGPTL2 or sh-NC. (B) The levels of TGF- $\beta 1$, Collagen IV, PTEN, and FN in control rats, DN rats, or DN rats infected with sh-ANGPTL2 or sh-NC were determined by western blot. (C) Representative images $(\times 400$ magnification) of WT1-positive staining. WT1-positive cells were counted in at least 10

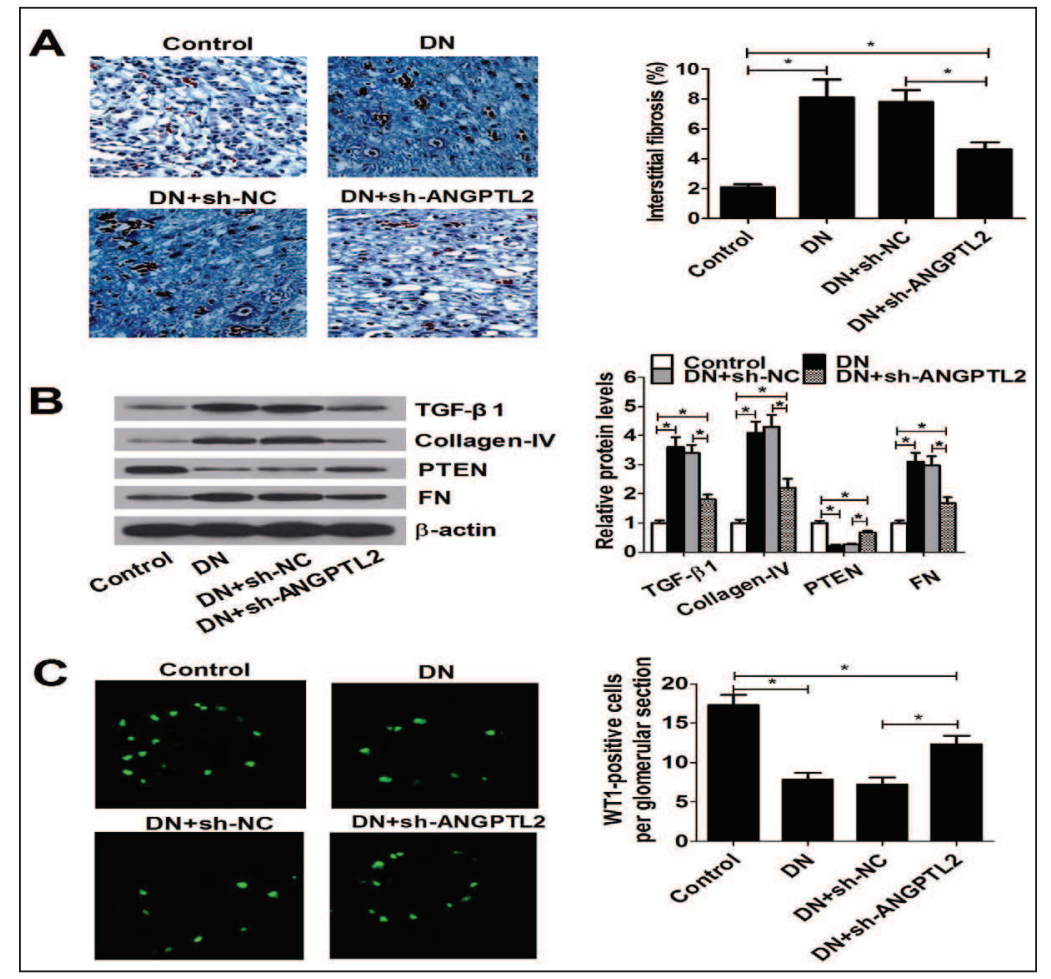
random glomerular sections. The data was expressed as mean $\pm \mathrm{SD}, \mathrm{n}=3, * \mathrm{P}<0.05$. Two-way ANOVA with Bonferroni test was used.

markers Wilms' tumor-1 protein (WT-1) and podocin expressions were down-regulated in DN rats, which were partially reversed after ANGPTL2 knockdown (Fig. 2A), indicating the involvement of ANGPTL2 in DN. Subsequently, body weight, 24-h proteinuria, blood glucose level, serum creatinine and blood urea nitrogen were measured. The body weight in DN rats was markedly lower than that in control group, while ANGPTL2 knockdown substantially restored body weight compared with sh-NC group in DN rats $(P<0.05)$ (Fig. 2B). As displayed in Fig. 2C-2F, the concentrations of 24-h proteinuria, blood glucose level, serum creatinine and blood urea nitrogen were all increased in DN rats compared with control group, whereas these effects were weakened following ANGPTL2 knockdown in contrast to sh-NC group $(P<$ 0.05). Taken together, these data revealed that ANGPTL2 knockdown relieved renal damage of STZ-induced DN rats.

ANGPTL2 knockdown mitigated STZ-induced inflammatory response in DN rats

To examine the role of ANGPTL2 in the regulation of inflammatory cytokines in DN rats, we further analyzed the concentrations of IL- 6 , IL- $1 \beta$, TNF- $\alpha$, and MCP- 1 in blood and kidney cortex of DN rats by ELISA. The results manifested that the levels of IL-6 (Fig. 3A and 3E), IL$1 \beta$ (Fig. 3B and 3F), TNF- $\alpha$ (Fig. 3C and 3G), and MCP-1 (Fig. 3D and 3H) in blood and kidney cortex of DN rats were all increased compared with the control group. By contrast, the secretion of the inflammatory cytokines was remarkably inhibited by ANGPTL2 knockdown compared to sh-NC group in DN rats $(P<0.05)$. Taken together, these findings suggested that ANGPTL2 knockdown suppressed STZ-induced inflammatory cytokines generation in DN rats.

ANGPTL2 knockdown attenuated STZ-induced renal fibrosis and ECM accumulation

To investigate the effect of ANGPTL2 knockdown on renal histology of DN, the pathologic characteristics associated with DN in glomeruli were examined in STZ-induced DN rat model. As shown in Fig. 4A, histological examination of kidney sections staining with Masson's 


\section{Cellular Physiology Cell Physiol Biochem 2017;43:685-696

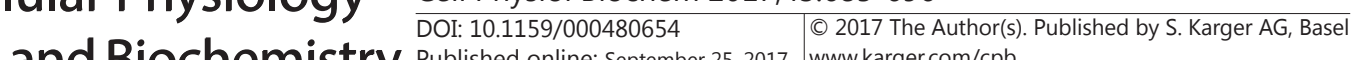 and Biochemistry Published online: September 25, 2017 www.karger.com/cpb}

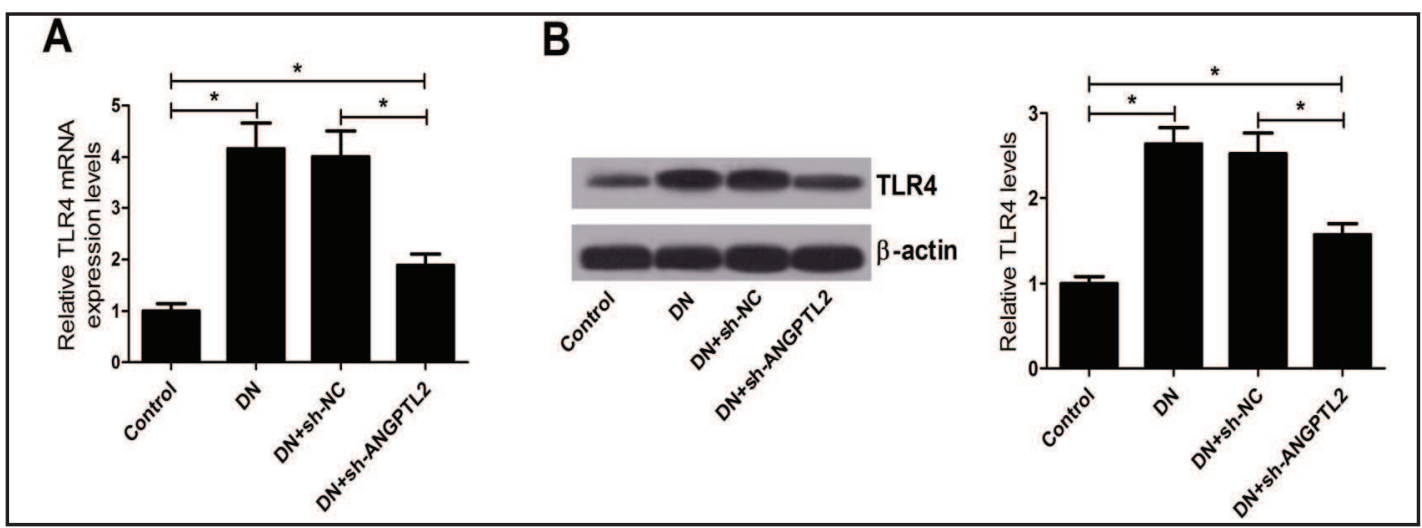

Fig. 5. Effect of ANGPTL2 knockdown TLR4 expression in DN rats. The expression of TLR4 at mRNA (A) and protein (B) levels was determined by qRT-PCR and western blot, respectively. The data was expressed as mean $\pm \mathrm{SD}, \mathrm{n}=3, * \mathrm{P}<0.05$. Two-way ANOVA with Bonferroni test was used.

Fig. 6. Effect of ANGPTL2 knockdown on the expressions of inflammatory cytokines in HG-induced podocytes. The expressions of IL-6 (A), IL-1 $\beta$ (B), TNF- $\alpha$ (C), and MCP-1 (D) mRAN in control cells, HG-stimulated podocytes, and HG-stimulated podocytes transfected with si-ANGPTL2 or si-NC were assessed by qRT-PCR. The data was expressed as mean $\pm \mathrm{SD}, \mathrm{n}=3,{ }^{*} \mathrm{P}<0.05$. Two-way ANOVA with Bonferroni test was used.

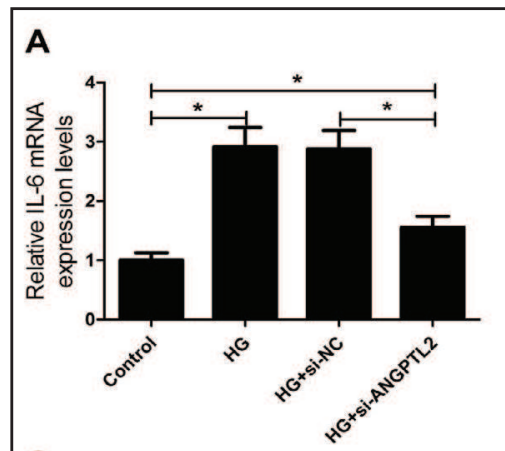

C

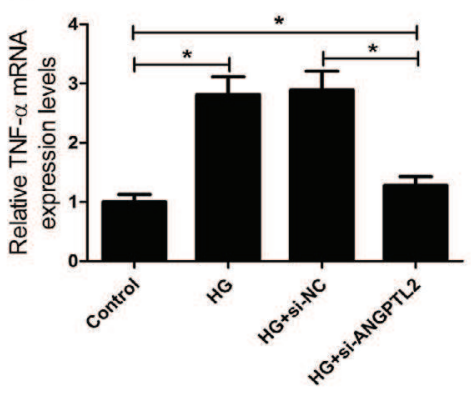

B

D
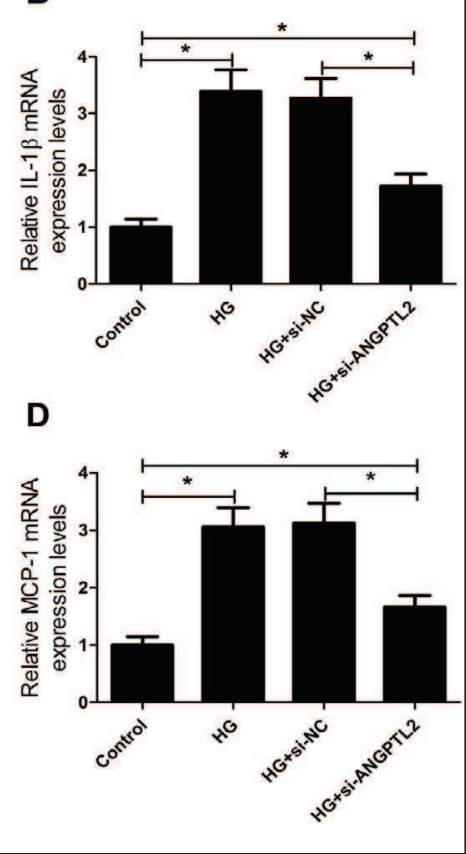

trichrome implied a remarkable accumulation of collagen in the kidney of STZ-induced DN rats compared with control group, suggesting the development of interstitial fibrosis. On the contrary, ANGPTL2 knockdown markedly inhibited the collagen accumulation and interstitial fibrosis $(P<0.05)$ in DN rats. It was reported that decreased expression of PTEN in mesangial cells contributed to expansion of the mesangial matrix [20]. Thus, PTEN expression in DN rats was determined to evaluate the accumulation of ECM. As displayed in Fig. 4B, TGF- $\beta 1$, a fibrosis-inducing protein in glomeruli, as well as two major ECM protein, Collagen IV and fibronectin (FN), were significantly highly expressed, and PTEN expression was down-regulated in DN rats compared with control group, while ANGPTL2 knockdown dramatically reversed these effects $(P<0.05)$, indicating that ANGPTL2 knockdown abated the accumulation of ECM in DN rats. Additionally, the number of WT1-positive cells per glomerular section was decreased in DN rats compared with control group, while ANGPTL2 knockdown significantly increased the glomerular WT1-positive cell number versus sh-NC group $(P<0.05)$ (Fig. 4C), suggesting that ANGPTL2 knockdown was able to prevent podocyte 


\section{Cellular Physiology \\ Cell Physiol Biochem 2017;43:685-696 \begin{tabular}{l|l|l|l} 
DOI: 10.1159/000480654 & (C) 2017 The Author(s). Published by S. Karger AG, Basel
\end{tabular}

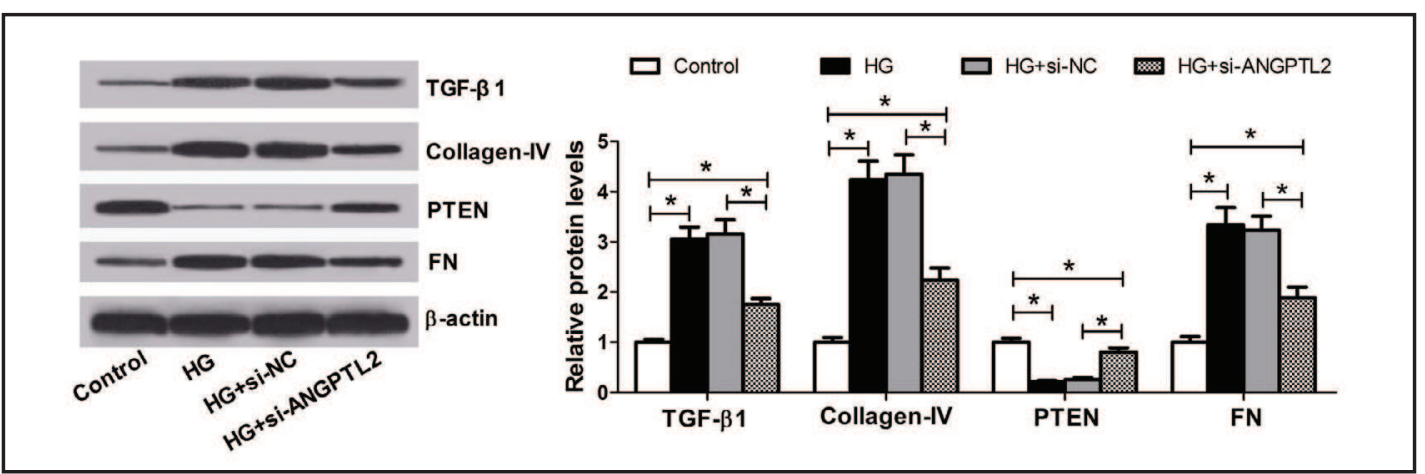

Fig. 7. Effect of ANGPTL2 knockdown on ECM accumulation in HG-induced podocytes. Western blot was performed to detect the levels of TGF- $\beta 1$, Collagen-IV, PTEN and FN in control cells, HG-stimulated podocytes, and HG-stimulated podocytes transfected with si-ANGPTL2 or si-NC. The data was expressed as mean $\pm \mathrm{SD}, \mathrm{n}=3,{ }^{*} \mathrm{P}<0.05$. Two-way ANOVA with Bonferroni test was used.

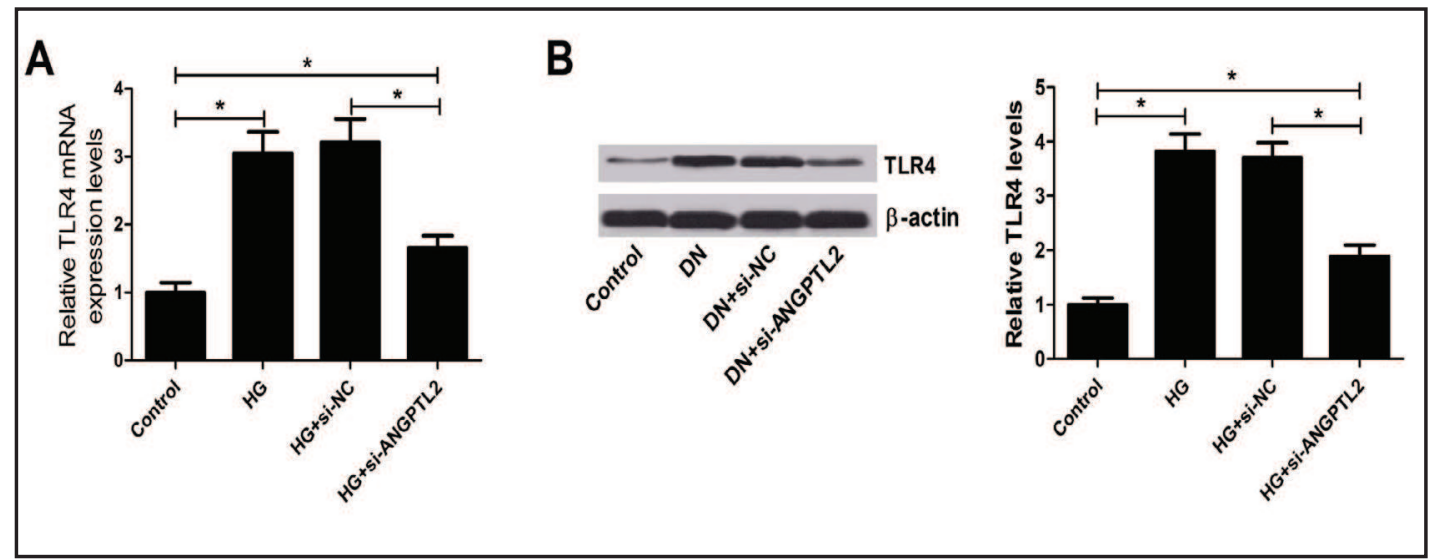

Fig. 8. Effect of ANGPTL2 knockdown on TLR4 expression in HG-induced podocytes. The expression of TLR4 at mRNA and protein levels in control cells, HG-stimulated podocytes, and HG-stimulated podocytes transfected with si-ANGPTL2 or si-NC was examined by RT-PCR and western blot. The data was expressed as mean $\pm S D, n=3, * P<0.05$. Two-way ANOVA with Bonferroni test was used.

loss. These results demonstrated that ANGPTL2 knockdown attenuated STZ-induced renal fibrosis, ECM accumulation, and prevented podocyte loss.

\section{ANGPTL2 knockdown inhibited TLR4 expression level in STZ-induced DN rats}

In order to explore the effect of ANGPTL2 on TLR4, the expression level of TLR4 at mRNA and protein levels in DN rats injected with sh-ANGPTL2 or sh-NC was evaluated by qRTPCR and western blot, respectively. As presented in Fig. 5A and 5B, the mRNA and protein levels of TLR4 in DN rats were both higher than that in control group, however, this effect was significantly abolished after ANGPTL2 knockdown $(P<0.05)$. All these data suggested that ANGPTL2 knockdown restrained TLR4 expression at mRNA and protein levels in STZinduced DN rats.

ANGPTL2 knockdown inhibited the levels of inflammatory cytokines in podocytes exposed to HG condition

The cell models of DN were established by treating podocytes with high glucose ( $30 \mathrm{mM})$ for $24 \mathrm{~h}$. We further analyzed the effect of ANGPTL2 knockdown on inflammatory cytokines expression in HG-induced podocytes. A significant increase in expressions of IL-6 (Fig. 6A), IL-1 $\beta$ (Fig. 6B), TNF- $\alpha$ (Fig. 6C), and MCP-1 (Fig. 6D) was observed in podocytes under HG 


\section{Cellular Physiology \\ Cell Physiol Biochem 2017;43:685-696 \begin{tabular}{ll|l} 
and Biochemistry & DOI: 10.1159/000480654 & $\begin{array}{l}\text { P 2017 The Author(s). Published by S. Karger AG, Basel } \\
\text { www.karger.com/cpb }\end{array}$ \\
\cline { 2 - 3 }
\end{tabular} \\ Yang et al.: ANGPTL2 Knockdown Ameliorates Diabetic Nephropathy}

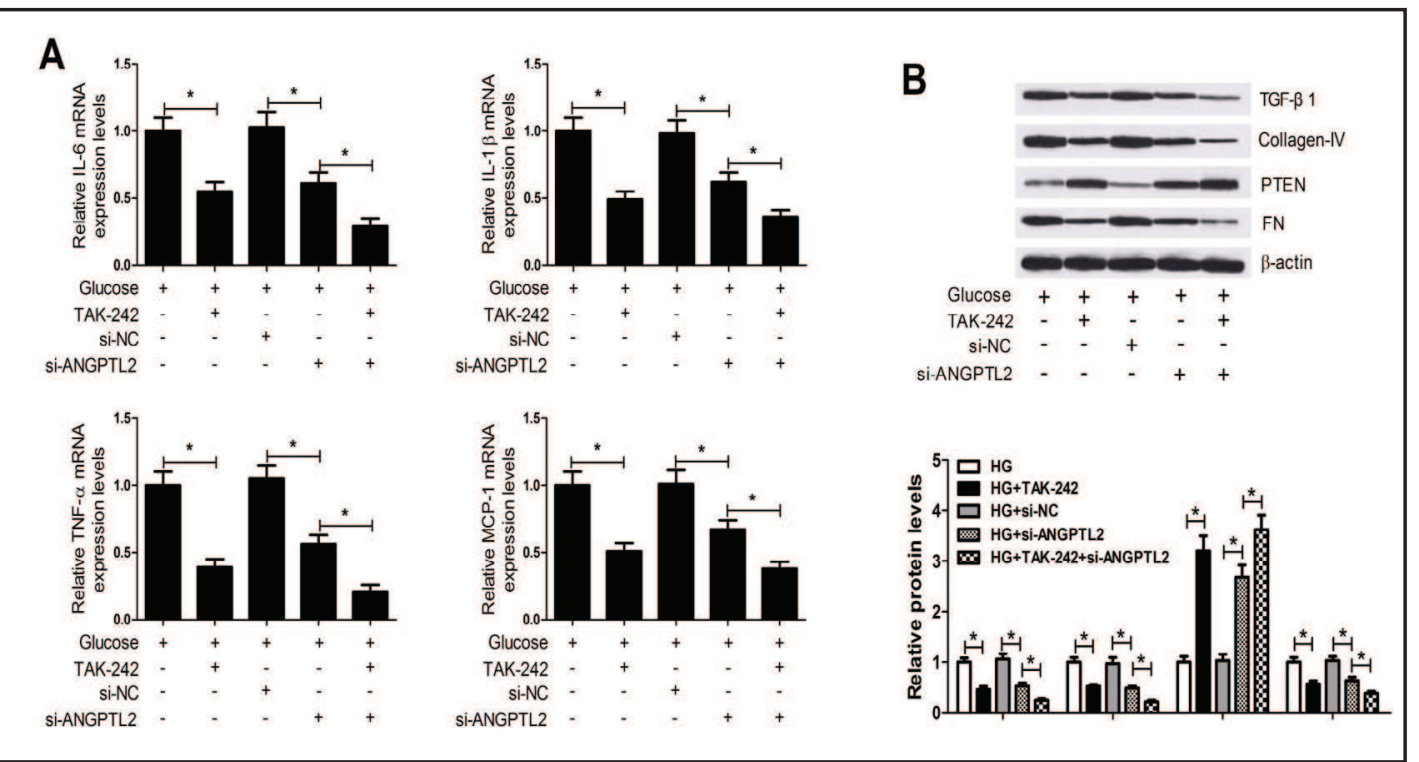

Fig. 9. Effect of TAK-242 treatment combined with ANGPTL2 knockdown on inflammatory cytokines secretion and ECM accumulation in HG-induced podocytes. Non-transfected or transfected (si-NC or siANGPTL2) podocytes were pretreated with or without $1 \mu \mathrm{M}$ TAK-242 for $2 \mathrm{~h}$, followed by stimulated with $30 \mathrm{mM}$ glucose for $24 \mathrm{~h}$. (A) The expressions of inflammatory cytokines including IL- 6 , IL-1 $\beta$, TNF- $\alpha$, and MCP-1 in treated podocytes were evaluated by qRT-PCR. (B) The levels of TGF- $\beta 1$, Collagen-IV, PTEN and FN in treated podocytes were determined by western blot. The data was expressed as mean $\pm S D, n=3,{ }^{*} P<0.05$. Two-way ANOVA with Bonferroni test was used.

conditions. However, siRNA-mediated ANGPTL2 knockdown led to an obvious suppression on inflammatory cytokines expression in HG-induced podocytes. Our data revealed that ANGPTL2 knockdown repressed HG-induced inflammatory cytokine expressions in podocytes.

\section{ANGPTL2 knockdown suppressed HG-induced ECM accumulation in podocytes}

To verify the effect of ANGPTL2 knockdown on ECM accumulation in vitro, the protein levels of TGF- $\beta 1$, Collagen-IV, PTEN and FN in HG-induced podocytes transfected with siANGPTL2 or si-NC were determined by western blot. As presented in Fig. 7, HG treatment improved TGF- $\beta 1$, Collagen-IV, and FN expression, while reduced PTEN level in podocytes. As expected, ANGPTL2 knockdown by si-ANGPTL2 dramatically counteracted these effects. These results indicated that ANGPTL2 knockdown diminished HG-induced ECM accumulation in podocytes.

ANGPTL2 knockdown inhibited TLR4 expression in podocytes exposed to HG conditions

The impact of ANGPTL2 knockdown on the expression level of TLR4 at mRNA and protein levels was further verified in HG-induced podocytes. As might be expected, qRT-PCR and western blot results disclosed that HG treatment strikingly promoted the expression of TLR4 at mRNA (Fig. 8A) and protein (Fig. 8B) levels in podocytes, but this effect was greatly abrogated after down-regulating ANGPTL2 by si-ANGPTL2 in HG-induced podocytes, confirming that ANGPTL2 knockdown repressed HG-induced TLR4 expression at mRNA and protein levels in podocytes.

ANGPTL2 knockdown suppressed inflammatory cytokines secretion and ECM accumulation by inhibiting TLR4 in HG-induced podocytes

TAK-242, a novel TLR4 antagonist, was administrated to investigate the underlying mechanism ANGPTL2 involved in podocytes under HG conditions. As demonstrated by qRT- 


\section{Cellular Physiology Cell Physiol Biochem 2017;43:685-696 and Biochemistry Published online: September 25, 2017 $\begin{aligned} & \text { D 2017 The Authors } \\ & \text { www.karger.com/cpb }\end{aligned}$}

Yang et al.: ANGPTL2 Knockdown Ameliorates Diabetic Nephropathy

PCR, the expressions of inflammatory cytokines including IL-6, IL-1 $\beta$, TNF- $\alpha$, and MCP-1 were substantially inhibited in TAK-242- or si- ANGPTL2-treated podocytes (Fig. 9A). More importantly, TAK-242 treatment significantly aggravated the inhibitory effect of ANGPTL2 knockdown on inflammatory cytokines expression. In addition, TAK-242 treatment or ANGPTL2 knockdown resulted in a remarkable reduction in the levels of TGF- $\beta 1$, Collagen-IV, and FN, and a significant improvement of PTEN level in podocytes exposed to HG conditions (Fig. 9B). Meanwhile, TAK-242 exacerbated the inhibitory effect of ANGPTL2 knockdown on ECM accumulation. Taken together, these results uncovered that ANGPTL2 knockdown ameliorated DN by inhibiting inflammatory cytokines secretion and ECM accumulation via down-regulating TLR4 in HG-induced podocytes.

\section{Discussion}

In the present study, we demonstrated that ANGPTL2 knockdown exerted a renoprotective effect via alleviating renal injury, inflammatory response, renal fibrosis and ECM accumulation in DN experimental model. Furthermore, our study suggested that the mitigative effects of ANGPTL2 knockdown on DN were mediated by inhibiting TLR4 under diabetic conditions.

ANGPTL2 has been identified as a mediator of the inflammatory process via direct activation of peritoneal monocytes and macrophages [21]. ANGPTL2 induced chronic inflammation of adipose tissue in the endothelial cells [8]. It was also reported that up-regulation of ANGPTL2 in diabetic glomerulopathy was implicated in abnormal microvasculature and endothelial inflammation [12]. Accordingly, elevated serum level of ANGPTL2 is positively associated with the development of type 2 diabetes [22]. More interestingly, elevated ANGPTL2 expression is independently associated with albuminuria, a significant clinical hallmark of early stage DN, suggesting a possible role of ANGPTL2 in progressive nephropathy with type 2 diabetes [12]. Additionally, histological analysis of human kidneys indicated the abundant expression of ANGPTL2 in renal tubule epithelial cells during progression of renal fibrosis, and ANGPTL2 deficiency reduced renal fibrosis by suppressing TGF- $\beta 1$ in kidney [23]. In line with the previous findings, our studies verified the up-regulation of ANGPTL2 in DN rats. Further function analysis revealed that adenovirus shRNA-mediated ANGPTL2 knockdown ameliorated DN by alleviating renal injury, inflammatory response, renal fibrosis and ECM accumulation in STZ-induced DN rats and HG-induced podocytes. Similarly, ANGPTL4, another member of ANGPTL family, was reported to be up-regulated and closely associated with albuminuria in STZ-induced diabetic rats and patients with DN [24].

Emerging evidence has indicated that TLR4 participated in the activation of the innate immune response by triggering a signaling cascade with the release of several inflammatory cytokines [25]. Recently, TLR4 is found to be vigorously associated with the pathogenesis of renal disorders including DN in a sterile environment [25]. Besides, it is well documented that TLR4 establishes a link between inflammation and fibrosis in chronic kidney diseases [26]. For example, Liu et al. demonstrated that TLR4 played a vital role in the occurrence and the development of DN by activating immune-inflammatory reactions [27]. Ma et al. reported that HG directly activated TLR4 in podocytes and tubular epithelial cells, inducing inflammation, podocyte and tubular epithelial cell injury and interstitial fibrosis [28]. STZinduced diabetic and uninephrectomized TLR4-deficient mice displayed less albuminuria, renal dysfunction interstitial and macrophage infiltration, implying that TLR4 may promote tubulointerstitial inflammation in DN [29]. Of note, a previous study hypothesized that TLR4 could be a potential receptor for ANGPTL2 in endothelial cells and monocytes, since ANGPTL2 possessed a fibrinogen-like domain at the C-terminus and fibrinogen acted as an intrinsic TLR4 ligand [18]. In our study, TLR4 was demonstrated to be highly expressed at mRNA and protein levels in STZ-induced DN rats and HG-induced podocytes, which was significantly suppressed after ANGPTL2 knockdown. Moreover, either TAK-242 treatment or ANGPTL2 


\section{Cellular Physiology Cell Physiol Biochem 2017;43:685-696

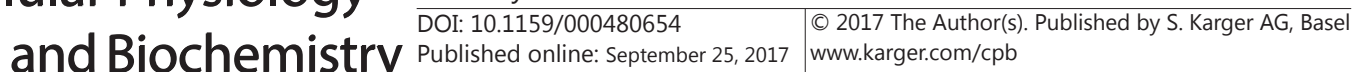

Yang et al.: ANGPTL2 Knockdown Ameliorates Diabetic Nephropathy

knockdown suppressed the production of inflammatory cytokines and accumulation of ECM. Furthermore, the inhibitory effect of ANGPTL2 knockdown inflammatory cytokines expression and ECM accumulation was exacerbated after treatment with TAK-242 in HGinduced podocytes, suggesting that ANGPTL2 knockdown ameliorated DN by suppressing inflammatory cytokines secretion and ECM accumulation via down-regulating TLR4.

In conclusion, we confirmed that expression of ANGPTL2 and TLR4 was up-regulated in DN rats. ANGPTL2 knockdown ameliorated DN by alleviating renal injury, inflammatory response, renal fibrosis and ECM accumulation in STZ-induced DN rat model or HG-induced podocytes. Mechanistic studies revealed that ANGPTL2 knockdown exerted its protective effect on DN by inhibiting TLR4 expression in vitro, contributing to a better understanding of the pathogenetic mechanism of DN and providing potential therapeutic target for DN. However, the molecular correlation between ANGPTL2 and TLR4 suggested in the in vivo model of DN still need further investigation.

\section{Disclosure Statement}

The authors of this manuscript state that they have no Disclosure Statement to declare.

\section{References}

1 Schernthaner G, Schernthaner GH: Diabetic nephropathy: new approaches for improving glycemic control and reducing risk. J Nephrol 2013; 26:975-985.

$\longrightarrow 2$ Lei CT, Wei YH, Tang H, Wen Q, Ye C, Zhang C, Su H: PKC- $\alpha$ triggers EGFR ubiquitination, endocytosis and ERK activation in podocytes stimulated with high glucose. Cell Physiol Biochem 2017; 42:281-294.

- 3 Hu C, Sun L, Xiao L, Han Y, Fu X, Xiong X, Xu X, Liu Y, Yang S, Liu F, Kanwar YS: Insights into the Mechanisms Involved in the Expression and Regulation of Extracellular Matrix Proteins in Diabetic Nephropathy. Curr Med Chem 2015; 22:2858-2870.

4 Tuttle KR: Linking metabolism and immunology: diabetic nephropathy is an inflammatory disease. J Am Soc Nephrol 2005; 16:1537-1538.

-5 Reddy MA, Tak Park J, Natarajan R: Epigenetic modifications in the pathogenesis of diabetic nephropathy. Semin Nephrol 2013; 33:341-353.

6 Huang H, You Y, Lin X, Tang C, Gu X, Huang M, Qin Y, Tan J, Huang F: Inhibition of TRPC6 signal pathway alleviates podocyte injury induced by TGF- $\beta 1$. Cell Physiol Biochem 2017; 41:163-172.

7. Kim I, Moon SO, Koh KN, Kim H, Uhm CS, Kwak HJ, Kim NG, Koh GY: Molecular cloning, expression, and characterization of angiopoietin-related protein. J Biol Chem 1999; 274:26523-26528.

8 Tabata M, Kadomatsu T, Fukuhara S, Miyata K, Ito Y, Endo M, Urano T, Zhu HJ, Tsukano H, Tazume H, Kaikita K, Miyashita K, Iwawaki T, Shimabukuro M, Sakaguchi K, Ito T, Nakagata N, Yamada T, Katagiri H, Kasuga M, Ando Y, Ogawa H, Mochizuki N, Itoh H, Suda T, Oike Y: Angiopoietin-like protein 2 promotes chronic adipose tissue inflammation and obesity-related systemic insulin resistance. Cell Metab 2009; 10:178-188.

9 Thorin-Trescases N, Thorin E: Angiopoietin-like-2: a multifaceted protein with physiological and pathophysiological properties. Expert Rev Mol Med 2014; 16:e17.

10 Farhat N, Thorin-Trescases N, Mamarbachi M, Villeneuve L, Yu C, Martel C, Duquette N, Gayda M, Nigam A, Juneau M, Allen BG, Thorin E: Angiopoietin-like 2 promotes atherogenesis in mice. J Am Heart Assoc 2013; 2:e000201.

11 Huang CL, Wu YW, Wu CC, Hwang JJ, Yang WS: Serum Angiopoietin-Like Protein 2 Concentrations Are Independently Associated with Heart Failure. PLoS One 2015; 10:e0138678.

12 Sun H, Zheng JM, Chen S, Zeng CH, Liu ZH, Li LS: Enhanced expression of ANGPTL2 in the microvascular lesions of diabetic glomerulopathy. Nephron Exp Nephrol 2007; 105:e117.

13 Wu H, Ma J, Wang P, Corpuz TM, Panchapakesan U, Wyburn KR, Chadban SJ: HMGB1 contributes to kidney ischemia reperfusion injury. J Am Soc Nephrol 2010; 21:1878-1890.

14 Beutler B: Inferences, questions and possibilities in Toll-like receptor signalling. Nature 2004; 430:257263. 


\section{Cellular Physiology Cell Physiol Biochem 2017;43:685-696 \begin{tabular}{ll|l} 
and Biochemistry & Published online: September 25, 2017 & $\begin{array}{l}\text { ( ) } 2017 \text { The Author(s). Published by S. Karger AG, Basel } \\
\text { www.karger.com/cpb }\end{array}$
\end{tabular}}

Yang et al.: ANGPTL2 Knockdown Ameliorates Diabetic Nephropathy

15 Devaraj S, Jialal I, Yun JM, Bremer A: Demonstration of increased toll-like receptor 2 and toll-like receptor 4 expression in monocytes of type 1 diabetes mellitus patients with microvascular complications. Metabolism 2011; 60:256-259.

16 Kaur H, Chien A, Jialal I: Hyperglycemia induces Toll like receptor 4 expression and activity in mouse mesangial cells: relevance to diabetic nephropathy. Am J Physiol Renal Physiol 2012; 303:F1145-1150.

17 Pulskens WP, Rampanelli E, Teske GJ, Butter LM, Claessen N, Luirink IK, van der Poll T, Florquin S, Leemans JC: TLR4 promotes fibrosis but attenuates tubular damage in progressive renal injury. J Am Soc Nephrol 2010; 21:1299-1308.

18 Oike Y, Tabata M: Angiopoietin-like proteins-potential therapeutic targets for metabolic syndrome and cardiovascular disease. Circ J 2009; 73:2192-2197.

19 Livak KJ, Schmittgen TD: Analysis of relative gene expression data using real-time quantitative PCR and the 2(-Delta Delta C(T)) Method. Methods 2001; 25:402-408.

20 Lin JS, Shi Y, Peng H, Shen X, Thomas S, Wang Y, Truong LD, Dryer SE, Hu Z, Xu J: Loss of PTEN promotes podocyte cytoskeletal rearrangement, aggravating diabetic nephropathy. J Pathol 2015; 236:30-40.

21 Umikawa M, Umikawa A, Asato T, Takei K, Matsuzaki G, Kariya K, Zhang CC: Angiopoietin-like protein 2 induces proinflammatory responses in peritoneal cells. Biochem Biophys Res Commun 2015; 467:235-241.

-22 Doi Y, Ninomiya T, Hirakawa Y, Takahashi O, Mukai N, Hata J, Iwase M, Kitazono T, Oike Y, Kiyohara Y: Angiopoietin-like protein 2 and risk of type 2 diabetes in a general Japanese population: the Hisayama study. Diabetes Care 2013; 36:98-100.

23 Morinaga J, Kadomatsu T, Miyata K, Endo M, Terada K, Tian Z, Sugizaki T, Tanigawa H, Zhao J, Zhu S, Sato M, Araki K, Iyama K, Tomita K, Mukoyama M, Tomita K, Kitamura K, Oike Y: Angiopoietin-like protein 2 increases renal fibrosis by accelerating transforming growth factor-beta signaling in chronic kidney disease. Kidney Int 2016; 89:327-341.

24 Ma J, Chen X, Li JS, Peng L, Wei SY, Zhao SL, Li T, Zhu D, He YX, Wei QJ, Li B: Upregulation of podocytesecreted angiopoietin-like-4 in diabetic nephropathy. Endocrine 2015; 49:373-384.

25 Yu R, Bo H, Villani V, Spencer PJ, Fu P: The Inhibitory Effect of Rapamycin on Toll Like Receptor 4 and Interleukin 17 in the Early Stage of Rat Diabetic Nephropathy. Kidney Blood Press Res 2016; 41:55-69.

-26 Lepenies J, Eardley KS, Kienitz T, Hewison M, Ihl T, Stewart PM, Cockwell P, Quinkler M: Renal TLR4 mRNA expression correlates with inflammatory marker MCP-1 and profibrotic molecule TGF-beta(1) in patients with chronic kidney disease. Nephron Clin Pract 2011; 119:c97-c104.

27 Liu SY, Nie XZ, Zhou WY, Chen J: Expression and effect of TLR4 in rats with diabetic nephropathy. Asian Pac J Trop Med 2013; 6:635-639.

28 Ma J, Chadban SJ, Zhao CY, Chen XC, Kwan T, Panchapakesan U, Pollock CA, Wu HL: TLR4 activation promotes podocyte injury and interstitial fibrosis in diabetic nephropathy. PLoS One 2014; 9: e97985.

29 Lin M, Yiu WH, Wu HJ, Chan LY, Leung JC, Au WS, Chan KW, Lai KN, Tang SC: Toll-like receptor 4 promotes tubular inflammation in diabetic nephropathy. J Am Soc Nephrol 2012; 23:86-102. 